\title{
The effects of multiple controlled ovarian hyperstimulation over a 2-year period on ovarian reserve and reactivity: a retrospective clinical study
}

\author{
Xiaomeng Bu, Yanmin Ma, Chanwei Jia, Yanjun Liu, Qiaoli Zhang, Shuyu Wang \\ Department of Reproductive Medicine, Beijing Obstetrics and Gynecology Hospital, Capital Medical University, Beijing, China \\ Contributions: (I) Conception and design: X Bu, S Wang; (II) Administrative support: Y Ma; (III) Provision of study materials or patients: Y Liu, Q \\ Zhang; (IV) Collection and assembly of data: X Bu, Y Liu; (V) Data analysis and interpretation: X Bu; (VI) Manuscript writing: All authors; (VII) \\ Final approval of manuscript: All authors. \\ Correspondence to: Shuyu Wang, MB. Department of Reproductive Medicine, Beijing Obstetrics and Gynecology Hospital, Capital Medical \\ University, No. 251 Yaojiayuan Road, Chaoyang District, Beijing 100026, China. Email: wangshuyu@ccmu.edu.cn.
}

Background: To evaluate the effects of controlled ovarian hyperstimulation $(\mathrm{COH})$ on ovarian reserve function during in vitro fertilization and embryo transfer (IVF-ET).

Methods: From August 2018 to August 2020, the medical records of patients who received IVF-ET in the Department of Reproductive Medicine, Beijing Gynaecology and Obstetrics Hospital, Capital Medical University were analyzed retrospectively. Among them, 372 patients received 2 cycles of COH, 54 patients received 3 cycles, and 13 patients received 4 cycles. The levels of follicle-stimulating hormone (FSH), the number of antral follicles, levels of anti-Müllerian hormone (AMH), the total amount of gonadotropin (GN), the time of ovulation induction, the number of eggs obtained, the number of available embryos, and the number of high-quality embryos cycles were compared in different treatment.

Results: The age of female patients did not significantly affect the levels of AMH or FSH during menstruation, nor the number of antral follicles before ovulation induction $(\mathrm{P}>0.05)$. However, with an increase in age, an increase in the number of controlled $\mathrm{COH}$ cycles was observed. In patients who underwent $2 \mathrm{COH}$ cycles, the number of high-quality embryos in the second cycle increased significantly compared to the first cycle $(\mathrm{P}<0.05)$. However, there were no significant differences in the ovulation induction time, the number of eggs, the GN dosage, and the number of available embryos $(\mathrm{P}>0.05)$. In patients with 3 treatment cycles, the GN dose used in the third cycle was significantly lower than that used in the first cycle $(\mathrm{P}<0.05)$. There were no significant differences in the ovulation induction time, the number of eggs obtained, and the quality of embryos $(\mathrm{P}>0.05)$. In patients with 4 treatment cycles, significant differences were observed in the ovulation induction time between the first and the fourth controlled $\mathrm{COH}$ cycle $(\mathrm{P}<0.05)$. However, no significant differences were detected in $\mathrm{GN}$ dosage, ovulation induction time, the number of eggs obtained, the number of available embryos, and the quality of embryos $(\mathrm{P}>0.05)$.

Conclusions: Ovarian reserve function was not significantly affected in patients with up to 4 ovarian stimulation cycles.

Keywords: In vitro fertilization-embryo transfer (IVF-ET); controlled ovarian hyperstimulation (COH); ovarian reserve function

Submitted Jan 11, 2021. Accepted for publication Apr 04, 2021.

doi: 10.21037/apm-21-330

View this article at: http://dx.doi.org/10.21037/apm-21-330 


\section{Introduction}

The use of assisted reproductive technology emerged nearly 40 years ago. In vitro fertilization and embryo transfer (IVF-ET) is a technology that uses assisted reproductive technology to transfer embryo combined with in vitro fertilization into the patient to achieve pregnancy. At present, the clinical pregnancy rate of a single IVF-ET event is $40-60 \%$, and the live birth rate can reach $20-30 \%$. However, there are still many infertile couples who cannot achieve clinical pregnancy after one round of IVF-ET (1). These patients usually receive multiple controlled ovarian hyperstimulation $(\mathrm{COH})$ treatments to improve the probability of pregnancy. However, the effects of multiple $\mathrm{COH}$ treatments and multiple ovarian punctures (necessary for follicle collection) on ovarian reserve function remains controversial $(2,3)$. Some researchers believe that repeated $\mathrm{COH}$ does not affect the ovarian reserve function, and by adjusting the treatment plan, high-quality embryo formation rates and clinical pregnancy rates are significantly increased after repeated $\mathrm{COH}$ treatments. Some scholars believe the use of high dose FSH may accelerate the consumption of follicle reserves and reduce ovarian reactivity (4).

In this study, the clinical data of patients who received multiple $\mathrm{COH}$ treatments were retrospectively analyzed to further clarify the changes in ovarian reserve function and its reactivity after multiple $\mathrm{COH}$ iterations. This information will provide a scientific basis for assessing the risks and benefits of repeated $\mathrm{COH}$ on female ovarian function.

We present the following article in accordance with the STROBE reporting checklist (available at http://dx.doi. org/10.21037/apm-21-330).

\section{Methods}

\section{Study population}

Medical records were collected from patients who received IVF/intracytoplasmic sperm injection (ICSI)-ET after 2-4 cycles of COH between January 2018 and August 2020.

The inclusion criteria were as follows: (I) female patients aged 20-40 years; (II) causes of infertility included fallopian tube factors, ovulation disorders, endometriosis, or female and/or male factors; and (III) the interval time of each $\mathrm{COH}$ treatment cycle was more than 3 months. The exclusion criteria were as follows: (I) the levels of follicle-stimulating hormone (FSH) were greater than 10 IU/L; (II) the number of antral follicles was less than 5; (III) the levels of anti-
Müllerian hormone (AMH) were less than $0.8 \mathrm{ng} / \mathrm{mL}$; and (IV) the interval time between the first $\mathrm{COH}$ and the last $\mathrm{COH}$ exceeded 2 years.

A total of 964 patient cycles of $\mathrm{COH}$ with complete data were included in this retrospective study. Among them, 375 patients were treated for 2 cycles of $\mathrm{COH}, 54$ patients had 3 cycles, and 13 patients underwent 4 cycles. Self-control comparison was performed for all patients (Figure 1).

The study was conducted in accordance with the Declaration of Helsinki (as revised in 2013) and was approved by institutional ethics board of Beijing Gynecology and Obstetrics Hospital, Capital Medical University (No.: 2016-KY-085-01). Individual consent for this retrospective analysis was waived.

\section{Research design}

\section{Therapeutic regimen}

The ovarian stimulation plan for each patient was determined according to the levels of FSH and AMH, and the number of antral follicles. The patient's response to medication at the previous ovulation induction was also considered. The ovarian stimulation plans included the gonadotropin-releasing hormone agonist plan (abbreviated as the agonist plan), the gonadotropin-releasing hormone antagonist plan (abbreviated as the antagonist plan), the luteal ovulation induction plan, and the micro-stimulation plan. When the diameter of 3 or more follicles measured $17 \mathrm{~mm}$ or greater, or when 2 or more follicles measured $18 \mathrm{~mm}$ or greater, the eggs were harvested by puncture under the guidance of transvaginal ultrasound after 36 hours using $250 \mathrm{mg}$ recombinant human chorionic gonadotropin injection (Elzer, Serono Europe) or $0.2 \mathrm{mg}$ triptorelin injection (Dabija, Huiling Pharmaceutical Co. Ltd.). The fertilization method was conventional IVF fertilization or ICSI fertilization.

\section{Evaluation of embryo quality}

After 16-18 hours of in vitro fertilization, the egg was examined under the dissecting microscope. Fertilized eggs with double pronucleus $(2 \mathrm{PN})$ were determined as normal fertilization. The quality of embryos in the cleavage stage was evaluated by observing the number and morphology of cleavage cells 3 days after collecting the eggs. The evaluation criteria for high-quality embryos were as follows: at least 8 -cell embryos 3 days after harvesting the eggs; blastocysts observed 5 days after collecting the eggs; and the fragmentation of cleavage balls was less than $10 \%$. The 


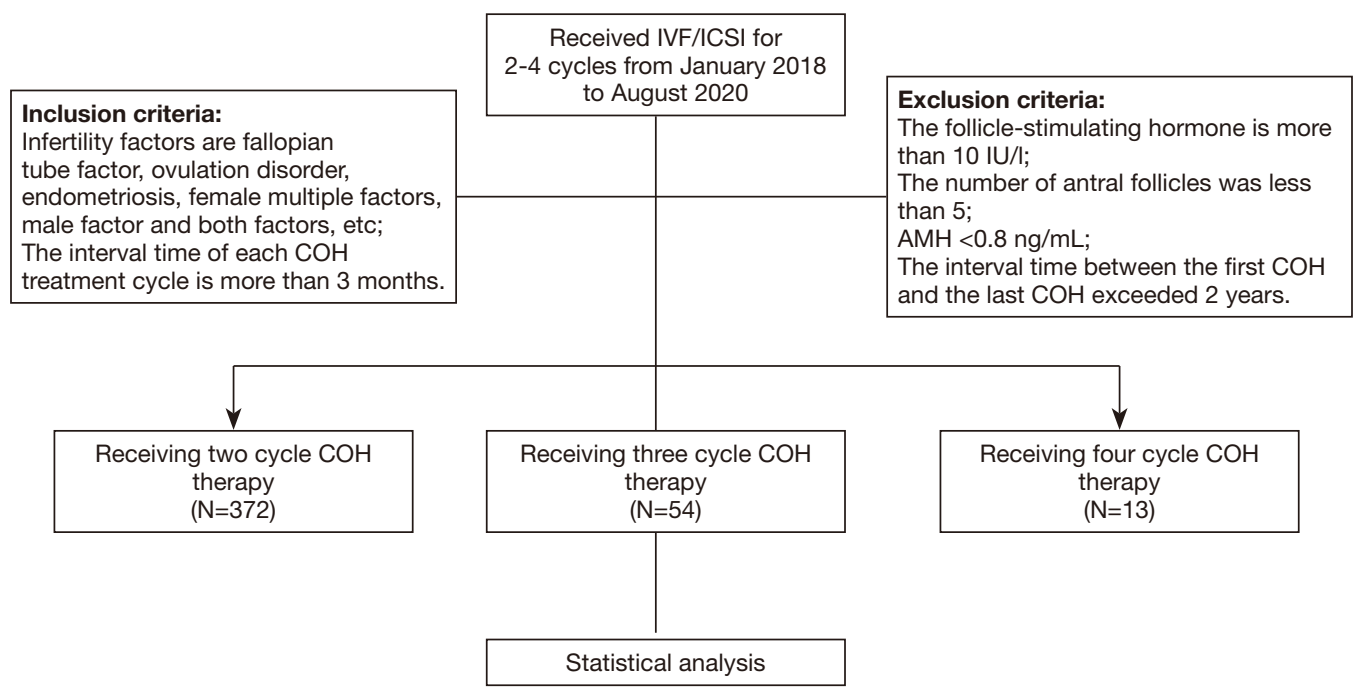

Figure 1 Flow diagram of the study design. AMH, anti-Müllerian hormone; $\mathrm{COH}$, controlled ovarian hyperstimulation.

numbers of available embryos and high-quality embryos were recorded.

\section{Observation indexes}

Basal FSH, the number of antral follicles, levels of AMH, GN dosage, ovulation induction time, the number of eggs obtained, the number of available embryos, and the number of high-quality embryos were assessed.

\section{Statistical analysis}

Data were expressed as mean \pm standard deviation for continuous variables and as proportions for categorical variables. The Student's $t$-test was used for pairwise comparison. Statistical analyses were performed using SPSS24.0 software. A P value $<0.05$ was considered statistically significant.

\section{Results}

A comparison of ovarian reserve function and reactivity in patients receiving 2 cycles of controlled $\mathrm{COH}$ therapy

A total of 372 patients received 2 cycles of $\mathrm{COH}$ therapy. There were no significant differences in the levels of $\mathrm{AMH}$, basal FSH, and antral follicle numbers before the start of each cycle $(\mathrm{P}>0.05$; Table 1$)$.

Compared with the first $\mathrm{COH}$ cycle, the number of high-quality embryos in the second cycle increased significantly $(\mathrm{P}<0.05)$, but there were no significant differences in the ovulation induction time, the number of eggs, the GN dosage, and the number of available embryos ( $>0.05$; Table 2).

\section{A comparison of ovarian reserve function and reactivity in patients receiving 3 cycles of controlled $\mathrm{COH}$ therapy}

A total of 54 patients received 3 cycles of $\mathrm{COH}$ therapy. There were no significant differences in the levels of $\mathrm{AMH}$, basal FSH levels, and antral follicle numbers before each cycle of ovulation induction ( $\mathrm{P}>0.05$; Table 3$)$.

Compared with the first $\mathrm{COH}$ cycle, the amount of GN in the third cycle was significantly lower $(\mathrm{P}<0.05)$. However, there were no significant differences in the ovulation induction time, the number of eggs obtained, and the quality of embryos $(\mathrm{P}>0.05$; Table 4$)$.

\section{A comparison of ovarian reserve function and reactivity in patients receiving four cycles of controlled $\mathrm{COH}$ therapy}

A total of 13 patients received 4 cycles of $\mathrm{COH}$ therapy, and no significant differences were found in the levels of $\mathrm{AMH}$, basal FSH levels, and antral follicle numbers before each cycle of ovulation induction $(\mathrm{P}>0.05$; Table 5$)$.

Significant differences were detected in the ovulation induction time between the first and the fourth cycles $(\mathrm{P}<0.05)$. However, there were no significant differences in GN dosage, ovulation induction time, the number of eggs obtained, the number of available embryos, and the quality 
Table 1 A comparison of ovarian reserve in women receiving two cycles of controlled ovarian hyperstimulation

\begin{tabular}{lcccc}
\hline Group & Age (year) & FSH $(\mathrm{mlU} / \mathrm{mL})$ & AMH $(\mathrm{ng} / \mathrm{nL})$ & AFC \\
\hline Cycle 1 & $32.14 \pm 5.21$ & $7.26 \pm 1.89$ & $6.23 \pm 5.87$ & $14.29 \pm 6.87$ \\
Cycle 2 & $32.65 \pm 3.59$ & $8 \pm 1.08$ & $4.11 \pm 2.12$ & $13.71 \pm 5.29$ \\
\hline
\end{tabular}

FSH, follicle-stimulating hormone; $\mathrm{AMH}$, anti-Müllerian hormone; AFC, antral follicle number.

Table 2 A comparison of ovarian reactivity in women receiving two cycles of controlled ovarian hyperstimulation

\begin{tabular}{cccccc}
\hline Group & GN usage (IU) & $\begin{array}{c}\text { Promotion time } \\
\text { (days) }\end{array}$ & $\begin{array}{c}\text { Number of eggs } \\
\text { obtained }\end{array}$ & $\begin{array}{c}\text { Number of embryos } \\
\text { available }\end{array}$ & $\begin{array}{c}\text { Number of high-quality } \\
\text { embryos }\end{array}$ \\
\hline Cycle 1 & $3,407 \pm 1,319.7$ & $12 \pm 2.65$ & $10.57 \pm 7.52$ & $2.71 \pm 1.38$ & $1.29 \pm 1.89$ \\
Cycle 2 & $3,437.5 \pm 1,342.83$ & $12 \pm 1.26$ & $13.57 \pm 7.98$ & $5.14 \pm 3.18$ & $3.29 \pm 3.2^{*}$ \\
\hline
\end{tabular}

Comparison with cycle $1{ }^{*} \mathrm{P}<0.05$. GN, gonadotropin.

Table 3 A comparison of ovarian reserve in women receiving three cycles of controlled ovarian hyperstimulation

\begin{tabular}{lcccc}
\hline Group & Age (year) & FSH $(\mathrm{mlU} / \mathrm{mL})$ & AMH $(\mathrm{ng} / \mathrm{nL})$ & AFC \\
\hline Cycle 1 & $34 \pm 2.55$ & $7.34 \pm 2.21$ & $4.42 \pm 2.85$ & $10.4 \pm 3.38$ \\
Cycle 3 & $35 \pm 1.63$ & $7.92 \pm 2.06$ & $3.28 \pm 2.01$ & $7.87 \pm 4.52^{*}$ \\
\hline
\end{tabular}

Comparison with cycle 1 * $\mathrm{P}<0.05$. FSH, follicle-stimulating hormone; $\mathrm{AMH}$, anti-Müllerian hormone; AFC, antral follicle number.

Table 4 A comparison of ovarian reactivity in women receiving three cycles of controlled ovarian hyperstimulation

\begin{tabular}{cccccc}
\hline Group & GN usage (IU) & $\begin{array}{c}\text { Promotion time } \\
\text { (day) }\end{array}$ & $\begin{array}{c}\text { Number of eggs } \\
\text { obtained }\end{array}$ & $\begin{array}{c}\text { Number of embryos } \\
\text { available }\end{array}$ & $\begin{array}{c}\text { Number of high-quality } \\
\text { embryos }\end{array}$ \\
\hline Cycle 1 & $3,285 \pm 1,572.68$ & $12.2 \pm 4.6$ & $9.4 \pm 7.6$ & $4.6 \pm 4.22$ & $0.2 \pm 0.45$ \\
Cycle 3 & $2,205 \pm 283.62^{*}$ & $11 \pm 3.24$ & $8.6 \pm 4.39$ & $2.6 \pm 1.34$ & $0.6 \pm 0.89$ \\
\hline
\end{tabular}

Comparison with cycle 1 * $\mathrm{P}<0.05$. GN, gonadotropin.

Table 5 A comparison of ovarian reserve in women receiving four cycles of controlled ovarian hyperstimulation

\begin{tabular}{lcccc}
\hline Group & Age (year) & FSH $(\mathrm{mlU} / \mathrm{mL})$ & AMH $(\mathrm{ng} / \mathrm{nL})$ & AFC \\
\hline Cycle 1 & $35 \pm 3.46$ & $7.52 \pm 1.44$ & $4.92 \pm 5.85$ & $12.67 \pm 9.81$ \\
Cycle 4 & $37 \pm 2.53$ & $8.31 \pm 2.2$ & $4.01 \pm 5.59$ & $10.33 \pm 7.5$ \\
\hline
\end{tabular}

$\mathrm{FSH}$, follicle-stimulating hormone; $\mathrm{AMH}$, anti-Müllerian hormone; $\mathrm{AFC}$, antral follicle number.

embryos $(\mathrm{P}>0.05$; Table 6).

\section{Discussion}

Ovarian reserve function refers to the ability of ovarian cortical follicles to grow and develop and into mature oocytes, which is manifested by the number of follicles and the quality of oocytes existing in the ovary (5). At present, the most commonly used indicators for evaluating ovarian reserve function include age, AMH levels, basal hormone endocrine levels including FSH, luteinizing hormone (LH) and estradiol (E2), and basal antral follicle numbers (6).

During $\mathrm{COH}$, the extra-physiological dose of GN can promote the growth and development of other follicles, avoid the occurrence of follicular atresia, and stimulate multiple oocytes. Some studies have shown that FSH 
Table 6 A comparison of ovarian reactivity in women receiving four cycles of controlled ovarian hyperstimulation

\begin{tabular}{cccccc}
\hline Group & GN usage (IU) & Promotion time (day) & $\begin{array}{c}\text { Number of eggs } \\
\text { obtained }\end{array}$ & $\begin{array}{c}\text { Number of embryos } \\
\text { available }\end{array}$ & $\begin{array}{c}\text { Number of high-quality } \\
\text { embryos }\end{array}$ \\
\hline Cycle 1 & $1,825 \pm 499.37$ & $8.67 \pm 1.15$ & $7.33 \pm 3.51$ & $3 \pm 1$ & $1.33 \pm 1.53$ \\
Cycle 4 & $2,100 \pm 75$ & $11 \pm 2.65^{\star}$ & $5.67 \pm 2.08$ & $3 \pm 1.73$ & $2.1 \pm 1.12$ \\
\hline
\end{tabular}

Comparison with cycle 1 * $\mathrm{P}<0.05$. GN, gonadotropin.

stimulation at super-physiological doses may promote the growth of the remaining follicles in the follicular pool and contribute to the recruited antral follicles, thus accelerating the depletion of the follicular pool (7).

In addition, it has been reported that repeated vaginal puncture of the ovary may lead to the release of ovarian autoantigens, resulting in a decrease in the number of follicles in the follicular pool (8). Other studies have shown that transvaginal follicular puncture may damage ovarian capillaries and peripheral ovarian tissues. This has a cumulative negative effect with each increasing number of punctures. Other reports have suggested that GN will change the physiological choice of a single dominant follicle, but will not accelerate the recruitment of follicles from the next cycle, indicating that repeated $\mathrm{COH}$ has no adverse effects on ovarian function (3).

Infertile women aged 20-40 years were selected for this study. The results demonstrated that there were no significant changes in the basal FSH and AMH levels between each cycle of $\mathrm{COH}$ in patients who received 2-4 cycles of $\mathrm{COH}$. This suggesting that $\mathrm{COH}$, up to 4 cycles, did not lead to any significant decrease in ovarian reserve function. However, in patients receiving 2-4 cycles of $\mathrm{COH}$ therapy, $\mathrm{AMH}$ gradually decreased with each ovarian stimulation cycles, and basal FSH gradually increased. The number of antral follicles also gradually decreased with the increase of ovarian stimulation cycles. Although there was no statistical significance in the above differences, with the increase of each $\mathrm{COH}$ cycle, the ovarian reserve function gradually decreased. It remains to be determined whether this phenomenon is caused by the increase in the age of the patients.

Ovarian reactivity refers to the response of the ovary to $\mathrm{GN}$ during $\mathrm{COH}$, and this is measured by GN dosage, ovulation induction time, number of eggs, and other indicators (9). At present, reports examining the effects of repeated $\mathrm{COH}$ on ovarian reactivity remain controversial.

Some researchers found that $25 \%$ of patients who used the antagonist regimen for repeated $\mathrm{COH}$ had increased
GN dosage and decreased ovarian reactivity, while $75 \%$ of patients had no obvious changes in ovarian reactivity (10). However, our current study showed that the GN dosage in the third cycle was lower than that in the first cycle among women who had undergone $\mathrm{COH}$ for 3 cycles. This may be related to the downward trend of the number of follicles in the basal sinus after 2 cycles of $\mathrm{COH}$ treatment, and this may have led to more doctors adopting micro-stimulation or less GN to promote ovulation. Among women who had undergone 4 cycles of $\mathrm{COH}$, the ovulation induction time in the fourth cycle was longer than that in the first cycle. This may be related to a decrease in ovarian reactivity.

It has been reported the antral follicle numbers in repeated $\mathrm{COH}$ cycles are significantly higher than that observed during the first cycle, and the number of eggs obtained, usable embryos, and high-quality embryos in repeated cycles are significantly higher than that in the first cycle (11). This study demonstrated that no significant changes were observed between any of the 4 cycles of $\mathrm{COH}$ in terms of the number of oocytes, usable embryos, and high-quality embryos obtained. However, the number of high-quality embryos tended to increase in the second, third, and fourth cycles. Previous reports have shown that adding growth hormone before and during ovulation promotion can improve the utilization rate of oocytes and improve the quality of embryos in $\mathrm{COH}$ treatment (12). In this study, 121 cases were pre-treated with growth hormone in the second cycle. The increase in the number of highquality embryos may be related to the increased use of growth hormone, adjustment of the medication plan by doctors, and the small number of samples.

In IVF treatment, most patients will receive large doses of GN stimulation and be subjected to repeated surgery for egg harvesting. Whether these treatments have a negative effect on ovarian reserve and responsiveness remains controversial $(7,13,14)$.

The results in this present study demonstrated that up to 4 cycles of $\mathrm{COH}$ treatment and egg harvesting did not significantly affect the ovarian reserve function. However, 
4 cycles of $\mathrm{COH}$ may lead to a degree of decline in ovarian responsiveness. The second $\mathrm{COH}$ cycle resulted in an increased number of high-quality embryos, while the third and fourth $\mathrm{COH}$ cycles also showed an increasing trend in the number of high-quality embryos. This may be related to the adjustment of the protocol and the appropriate application of growth hormone.

Further research into $\mathrm{COH}$ is warranted to optimize the number of high-quality embryos and therefore the number of successful pregnancies.

In summary, up to 4 cycles of $\mathrm{COH}$ treatment is safe, and reproductive physicians need to conduct a comprehensive evaluation of individual patients and select reasonable rowpromoting modes, drug doses, and pre-treatment modes to obtain a satisfactory outcome.

\section{Acknowledgments}

Funding: Beijing Hospitals Authority Clinical medicine Development of special funding support, code: XMLX202122.

\section{Footnote}

Reporting Checklist: The authors have completed the STROBE reporting checklist. Available at http://dx.doi. org/10.21037/apm-21-330

Data Sharing Statement: Available at http://dx.doi. org/10.21037/apm-21-330

Conflicts of Interest: All authors have completed the ICMJE uniform disclosure form (available at http://dx.doi. org/10.21037/apm-21-330). The authors have no conflicts of interest to declare.

Ethical Statement: The authors are accountable for all aspects of the work in ensuring that questions related to the accuracy or integrity of any part of the work are appropriately investigated and resolved. The study was conducted in accordance with the Declaration of Helsinki (as revised in 2013) and was approved by institutional ethics board of Beijing Gynecology and Obstetrics Hospital, Capital Medical University (No.: 2016-KY-085-01). Individual consent for this retrospective analysis was waived.

Open Access Statement: This is an Open Access article distributed in accordance with the Creative Commons
Attribution-NonCommercial-NoDerivs 4.0 International License (CC BY-NC-ND 4.0), which permits the noncommercial replication and distribution of the article with the strict proviso that no changes or edits are made and the original work is properly cited (including links to both the formal publication through the relevant DOI and the license). See: https://creativecommons.org/licenses/by-nc-nd/4.0/.

\section{References}

1. Wadhwa L, Mishra M. Therapeutic Efficacy of Endometrial Scratching in Repeated Controlled Ovarian Stimulation (COS) Failure Cycles. J Hum Reprod Sci 2018;11:59-71.

2. Norman RJ, Zegers-Hochschild F, Salle BS, et al. Repeated ovarian stimulation with corifollitropin alfa in patients in a GnRH antagonist protocol: no concern for immunogenicity. Hum Reprod 2011;26:2200-8.

3. Luk J, Arici A. Does the ovarian reserve decrease from repeated ovulation stimulations? Curr Opin Obstet Gynecol 2010;22:177-82.

4. Zhang H, Zheng W, Shen $\mathrm{Y}$, et al. Experimental evidence showing that no mitotically active female germline progenitors exist in postnatal mouse ovaries. Proc Natl Acad Sci U S A 2012;109:12580-5.

5. Aydogan Mathyk B, Aslan Cetin B, Bilici S, et al. Evaluation of ovarian reserve in women with psoriasis. Gynecol Endocrinol 2019;35:608-11.

6. Tal R, Seifer DB. Ovarian reserve testing: a user's guide. Am J Obstet Gynecol 2017;217:129-40.

7. Revelli A, Gennarelli G, Biasoni V, et al. The Ovarian Sensitivity Index (OSI) Significantly Correlates with Ovarian Reserve Biomarkers, Is More Predictive of Clinical Pregnancy than the Total Number of Oocytes, and Is Consistent in Consecutive IVF Cycles. J Clin Med 2020;9:1914.

8. Caligara C, Navarro J, Vargas G, et al. The effect of repeated controlled ovarian stimulation in donors. Hum Reprod 2001;16:2320-3.

9. Li M, Yao L, Xin M, et al. Dysregulation of collagen expression in peri-implantation endometrium of women with high ovarian response. J Obstet Gynaecol Res 2019;45:1035-44.

10. Rombauts L, Lambalk CB, Schultze-Mosgau A, et al. Intercycle variability of the ovarian response in patients undergoing repeated stimulation with corifollitropin alfa in a gonadotropin-releasing hormone antagonist protocol. Fertil Steril 2015;104:884-90.e2. 
11. Wang B, Kong N, Liu JY, et al. Repeated superovulation induction after failure in previous IVF-ET cycles with the ultra-long protocol: Analysis of outcomes of clinical pregnancy. Zhonghua Nan Ke Xue 2017;23:1099-102.

12. Cai MH, Liang XY, Wu YQ, et al. Six-week pretreatment with growth hormone improves clinical outcomes of poor ovarian responders undergoing in vitro fertilization treatment: A self-controlled clinical study. J Obstet Gynaecol Res 2019;45:376-81.

Cite this article as: $\mathrm{Bu} \mathrm{X,} \mathrm{Ma} \mathrm{Y,} \mathrm{Jia} \mathrm{C,} \mathrm{Liu} \mathrm{Y,} \mathrm{Zhang} \mathrm{Q,} \mathrm{Wang}$ $\mathrm{S}$. The effects of multiple controlled ovarian hyperstimulation over a 2-year period on ovarian reserve and reactivity: a retrospective clinical study. Ann Palliat Med 2021;10(4):4391-4397. doi: 10.21037/apm-21-330
13. Paul LT, Atilan O, Tulay P. The effect of repeated controlled ovarian stimulation cycles on the gamete and embryo development. Zygote 2019;27:347-9.

14. Al-Azemi M, Bernal AL, Steele J, et al. Ovarian response to repeated controlled stimulation in in-vitro fertilization cycles in patients with ovarian endometriosis. Hum Reprod 2000;15:72-5.

(English Language Editor: J. Teoh) 